\title{
LIDAR TRANSMITTER OFFERS “NON-DIFFRACTING" PROPERTY THROUGH SHORT DISTANCE IN HIGHLY-DENSE RANDOM MEDIA
}

Xiafukaiti Alifu*, Peng Ziqi and Tatsuo Shiina

\author{
Chiba University 1-33 Yayoi-cho, Inage-ku, Chiba-shi, Chiba 263-8522 JAPAN \\ *xapkat_alip@chiba-u.jp
}

\begin{abstract}
Non-diffracting beam (NDB) is useful in lidar transmitter because of its high propagation efficiency and high resolution. We aimed to generate NDB in random media such as haze and cloud. The laboratory experiment was conducted with diluted processed milk (fat: $1.8 \%, 1.1 \mu \mathrm{m} \varphi$ ). Narrow view angle detector of $5.5 \mathrm{mrad}$ was used to detect the forward scattering waveform. We obtained the central peak of NDB at the propagation distance of $5 \mathrm{~cm} \sim 30 \mathrm{~cm}$ in random media by adjusting the concentration of $<10 \%$.
\end{abstract}

\section{INTRODUCTION}

Aerosols and dusts have interactions between the ground and the cloud. They actively float in the low-altitude atmosphere. To observe them, we have developed an inline type micro-pulse lidar and a high-resolution polarization lidar, which have conducted polarization measurement in lowaltitude atmosphere [1-3]. The lidar transmitted beam catches strong scattering, hence it cannot penetrate at long distance in random media such as cloud and haze. Having a long distance penetration in random media is important for studying the remote sensing techniques such as deeper sensing in cloud structure and target recognition through haze [4-5].

In general, beams spread by its diffraction effect. However, in the case of a non-diffracting beam (NDB), such as Bessel beam, it can propagate over a long distance and keep its narrow beam width, because it does not diffract (self-formation) [6-8]. In previous study, it has been confirmed that an annular beam could change its beam shape into quasi-NDB after being scattered several times in random media (10$30 \mathrm{~cm}$ ) [9]. But, variation of NDB in different receiving distance was not clear. The objective of this research is to obtain variation of NDB in different receiving distances to analyze the components of the scattering waveform, and generate NDB at short distances $(5 \mathrm{~cm})$ in random media with higher concentration.

\section{ANNULAR BEAM}

In this research, the transmitted light of lidar is an annular beam, which is converted from a Gaussian beam by a pair of axicon prism. Figure 1 shows the transformation from the Gaussian beam to the annular beam using the axicon pair.

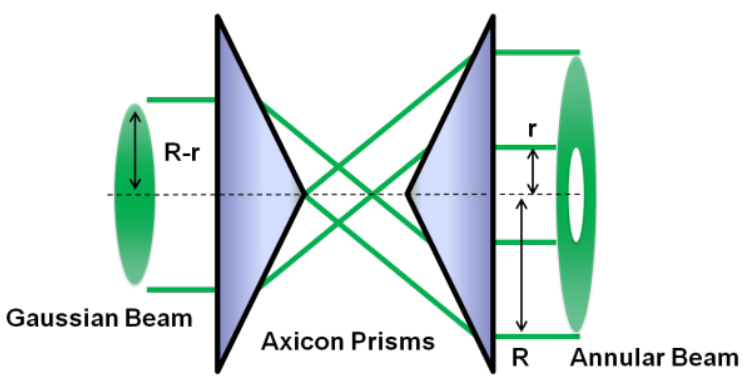

Figure 1 Transformation from the Gaussian beam to the annular beam using the axicon pair.

The Gaussian beam distribution $\mathrm{g}(\mathrm{r})$ is expressed in Eq.1.

$$
\mathrm{g}(r)=\frac{1}{\pi h^{2}} \exp \left[-\left(\frac{r}{h}\right)^{2}\right]
$$

Where $h$ is a half width at the point where the intensity equals $1 / \mathrm{e}^{2}$ of the central peak value, and $r$ represents radial point of the plane perpendicular to the propagation direction of light. The converted annular beam is expressed in Eq.2. $\mathrm{R}$ is the external radius of the annular beam.

$$
\mathrm{a}(r)=\sqrt{\frac{R-\mathrm{r}}{r}} g(R-r)
$$

In our laboratory experiment, we applied the annular beam as the lidar transmitted light, which has an external diameter of $40 \mathrm{~mm}$ with a ring 
width of $3 \mathrm{~mm}$. When the annular beam propagates in the atmosphere, as the propagation range increased, the energy is focused in the center due to a non-diffraction effect. Figure 2 shows the intensity distributions of the annular beam and NDB. This NDB was generated by propagating the annular beam at a long distance of $210 \mathrm{~m}$ in air, which is calculated by Fresnel equation. The center peak of NDB is narrow, and it has an advantage on optical sensing with high resolution.

\section{SYSTEM SETUP}

Figure 3 shows the experiment setup for the annular beam propagation through a random media. The experiment parameters are summarized in Table 1. A DPSS pulse laser with peak power of $4.6 \mathrm{~kW}, 532 \mathrm{~nm}$ wavelength, $2 \mathrm{~ns}$ pulse width and $10 \mathrm{kHz}$ repetition frequency was used. The ND filter was applied to adjust the intensity of the transmitted light. A diluted processed milk (milk fat: $1.8 \%, 1.1 \mu \mathrm{m} \varphi$ ) with $0 \%$ $\sim 6 \%$ concentration was used as a random media in this experiment. The media tanks of $20 \mathrm{~cm}(\mathrm{~W}) \times 20 \mathrm{~cm}(\mathrm{H}) \times 5 \mathrm{~cm} \sim 30 \mathrm{~cm}(\mathrm{~L})$ were used for the evaluating the propagation property of light in a short distance. The intensity distributions of the propagated light in random media were recorded by a receiving unit, which includes a collimating lens, a multimode optical fiber and a photomultiplier tube . This receiving unit had a narrow viewing angle of $5.5 \mathrm{mrad}$. The forward-scattered light in a random media is received selectively. The photomultiplier tube and a sampling oscilloscope were used to detect the weak signals from the media of definite concentration.

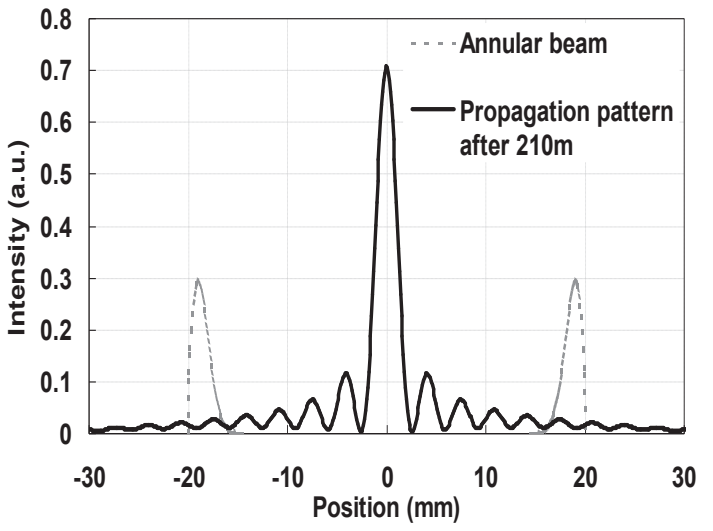

Figure 2 Intensity distributions of annular beam and its propagation after $210 \mathrm{~m}$.

Table 1 Parameters for the experimental setup

\begin{tabular}{|c|c|}
\hline Light source & $\begin{array}{l}\text { DPSS laser CryLas, 1Q532-1: } \\
\text { Wavelength: } 532 \mathrm{~nm} ; \\
\text { Peak power: } 4.6 \mathrm{~kW} ; \\
\text { Pulse Width: } 2 \mathrm{~ns} ; \\
\text { Repetition: } 10 \mathrm{kHz} \\
\end{array}$ \\
\hline $\begin{array}{c}\text { Annular beam } \\
\text { converter }\end{array}$ & $\begin{array}{l}\text { Axicon prims: } \\
\text { Zenith angle: } 150^{\circ}\left( \pm 10^{\prime}\right) \text {; } \\
\text { Core diameter: } 50.8 \mathrm{~mm} \text {; } \\
\text { Annular beam: } \\
\quad \text { External diameter : } 42 \mathrm{~mm}\end{array}$ \\
\hline $\begin{array}{l}\text { Random } \\
\text { media }\end{array}$ & $\begin{array}{l}\text { Media tank: } \\
\text { Material: tempax glass; } \\
\text { Size: } \mathrm{W} \times \mathrm{H} \times \mathrm{L} \\
\quad 20 \mathrm{~cm} \times 20 \mathrm{~cm} \times(1 \sim 30) \mathrm{cm} \text {; } \\
\text { Media: } \\
\text { Diluted processed milk: } \\
\quad \text { Milk fat: } 1.8 \% \text {; } \\
\quad \text { Fat size: } 1.1 \mu \mathrm{m} ;\end{array}$ \\
\hline Receiver & $\begin{array}{l}\text { Optical elements: } \\
\text { Optical Fiber: Multiple mode } \\
\text { Core Diameter: } 50 \mu \mathrm{m} ; \\
\text { Collimating lens: } \\
\text { Lens aperture: } 6 \mathrm{~mm} \\
\text { View angle: } 5.5 \mathrm{mrad} ;\end{array}$ \\
\hline
\end{tabular}

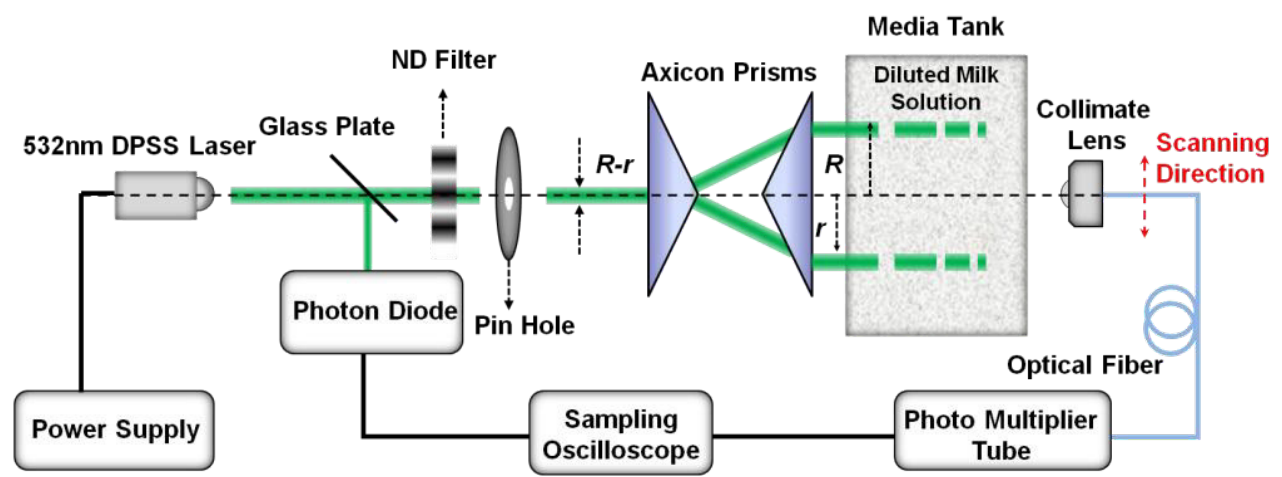

Figure 3 Experiment setup of annular light beam offers "non-diffracting” property through a random media. 


\section{RESULTS AND DISCUSSION}

When the annular beam propagated at $20 \mathrm{~cm}$ distance in milk diluted solution of $0.6 \%$ concentration, the forward scattering waveforms were measured at $5 \mathrm{~cm}$ and $10 \mathrm{~cm}$ away from the back of the media tank. Figure 4 shows the measured receiving waveforms. The center of the waveform has a peak $(6 \mathrm{~mm}$ in diameter) which we call NDB. The surrounding curve of the waveform is constructed by the multiple scattering lights (the intensity was normalized at position of $20 \mathrm{~mm}$ ). The central intensity ratio can be calculated by the ratio of the maximum intensities between NDB and multiple scattering lights. As a result, the central intensity ratio was $1.2 \%$ in both waveforms. Curvature radius of surrounding part of scattering waveforms was $9.6 \mathrm{~cm}$ when the receiving distance was $5 \mathrm{~cm}$. It changed to $10.5 \mathrm{~cm}$ and its intensity was declined overall when the receiving distance was $10 \mathrm{~cm}$. We confirmed that surrounding part of scattering waveforms spread out while center peak of NDB did not change its shape as the generated waveform propagated in air. Furthermore, according to the calculation, the generated waveform keeps its peak of NDB up to the propagation of $100 \mathrm{~cm}$ in the air. This result proves that the waveforms are composed of NDB in center part, which was constructed from forward scattering and the surrounding part, which was constructed from the multiple scattering, respectively.

To generate and control NDB in short-distance random media, we set the media tank of $5 \mathrm{~cm}$ and measured the scattering waveforms at the concentration range of $2 \% \sim 6 \%$. Figure 5 shows the measured waveforms at media concentration range of $3.5 \% \sim 5 \%$. At the beginning, a small peak appeared at the center of $3.5 \%$ concentration, the central intensity ratio became high as the concentration increases. The highest peak of NDB appeared at 5\% concentration, and the central intensity ratio was $1.8 \%$. In the previous study, the highest peak of NDB appeared at concentrations of $0.4 \%, 0.6 \%$ and $1 \%$ in the media tank of $30 \mathrm{~cm}, 20 \mathrm{~cm}$ and $10 \mathrm{~cm}$ respectively. But the highest peak of NDB appeared at the propagation distance of $5 \mathrm{~cm}$. In this case, the center intensity ratio was $50 \%$ higher than that at $20 \mathrm{~cm}$ propagation distance. Figure 6 shows the variation of the central intensity ratio in different

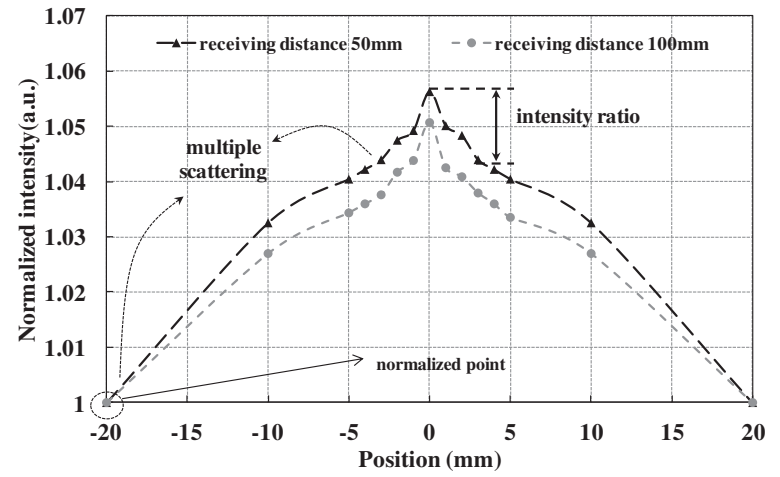

Figure 4 Scattering waveforms of annular beam through $20 \mathrm{~cm}$ long-distance random media by different receiving distance.

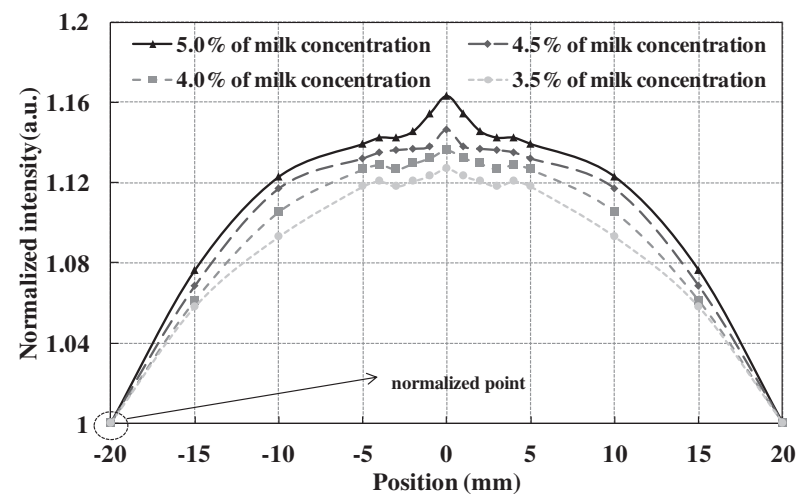

Figure 5 Scattering waveforms of annular beam at concentration range of $3.5 \% \sim 5 \%$ in random media by $5 \mathrm{~cm}$ propagation.

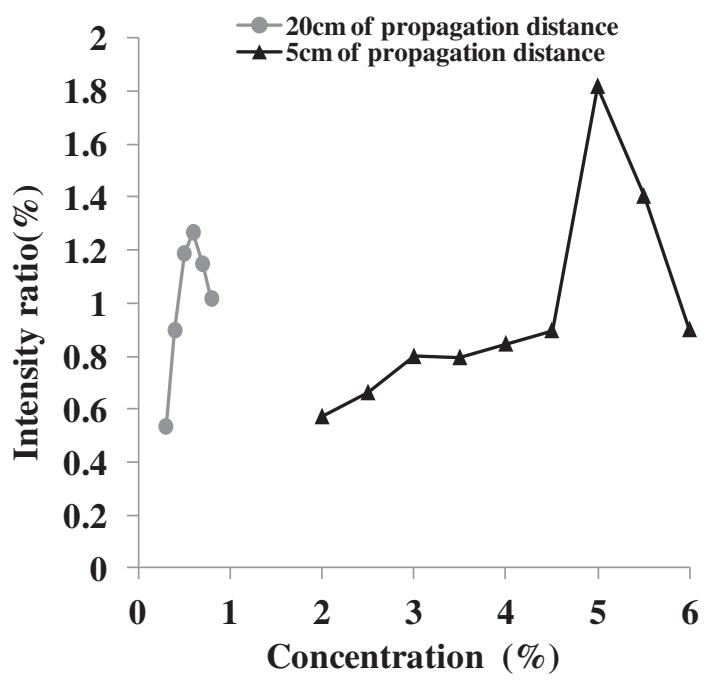

Figure 6 Analysis of intensity ratios at concentration increases by different propagation distance. 
concentration when the propagation distance is $5 \mathrm{~cm}$ and $20 \mathrm{~cm}$. For the case of $20 \mathrm{~cm}$ propagation, the NDB was generated at concentrations range of $0.3 \% \sim 0.8 \%$. The highest central intensity ratio of NDB was obtained at $0.6 \%$ concentration. The non-diffracting intensity start to decline when the concentration is higher than $0.6 \%$. In the case of $5 \mathrm{~cm}$ propagation, the NDB can be obtained at the concentration range of $2 \% \sim 6 \%$. The highest central intensity ratio of NDB was obtained at $5 \%$ concentration. Same to the case of $20 \mathrm{~cm}$ propagation, the non-diffracting intensity started to decline after this concentration in the $5 \mathrm{~cm}$ propagation. The concentration range of NDB appeared at propagation distance of $5 \mathrm{~cm}$ was 8times wider than that at propagation distance of $20 \mathrm{~cm}$. Since, the center part was subjected to stronger forward scattering at higher concentration in a short-distance random media. As a result, the non-diffracting field is offered the high center intensity in short distance [9].

\section{CONCLUSIONS}

This paper presents the propagation characteristics of NDB in different receiving distance to analyze the components of the scattering waveform and generation of NDB at short distances $(5 \mathrm{~cm})$ in random media with higher concentration. The experiment of different receiving distance demonstrated that NDB in center part is composed of forward scattering and surrounding part is composed of multiple scattering. According to the calculation, we have confirmed that the generated waveform keeps its peak of NDB up to the propagation distance of $100 \mathrm{~cm}$ in air. Moreover, we have successfully generated the NDB in the following condition; concentration of $2 \% \sim 6 \%$ and short-distance of $5 \mathrm{~cm}$ in random media. The highest center intensity ratio of $1.8 \%$ was obtained by high concentration media (5\%). By comparing the results from two different propagations $(5 \mathrm{~cm}$ and $20 \mathrm{~cm}$ ), the concentration range of existing NDB is wider at shorter propagation distance. The maximum intensity ratio of NDB is also higher in shorter distance, because the center part is subjected to stronger forward scattering in the higher concentration range media. This finding could be useful reference for more shorterdistance propagation $(<5 \mathrm{~cm})$ in future studies. In the future, the method which generates the NDB in random media by using annular beam can be applied for getting the deeper information of high concentration cloud and penetrating the heavy cloud as optical sensing field.

\section{References}

[1] Tatsuo Shiina, Eiji Minami, Masafumi Ito, and Yasuyuki Okamura, 2002: Optical circulator for an in-line-type compact lidar, Appl. Opt., Vol. 41, pp. 3900-3905

[2] Tatsuo Shiina, Kei Yoshida, Masafumi Ito, and Yasuyuki Okamura, 2005: In-line type micropulse lidar with an annular beam experiment, Appl. Opt., Vol. 44, pp. 7407-7413

[3] Tatsuo Shiina, Kazuo Noguchi, and Tetsuo Fukuchi, 2012: Polarization-independent optical circulator for high accuracy Faraday depolarization lidar, Appl. Opt., Vol. 51, pp. 898-904

[4] Michael I. Mishchenko, Janna M. Dlugach, Maxim A. Yurkin, Lei Bi, Brian Cairns, Li Liu, R. Lee Panetta, Larry D. Travis, Ping Yang, Nadezhda T. Zakharova 2016: First-principles modeling of electromagnetic scattering by discrete and discretely heterogeneous random media, Physics Reports, Vol.632, pp.1-75

[5] Yanhui $\mathrm{Li}$ and Zhensen $\mathrm{Wu}, 2010$ : Targets recognition using subnanosecond pulse laser range profiles, Opt. Express, Vol. 18, pp. 16788-16796

[6] J. Durnin and J. J. Miceli JR., 1987: Nondiffracting beams, Optics News, Vol. 13(12), pp. 27-27

[7] J. Durnin, J. H. Eberly, and J. J. Miceli, 1988: Comparison of Bessel and Gaussian beams, Opt. Lett., Vol. 13, pp. 79-80

[8] Tatsuo Shiina, Kei Yoshida, Masafumi Ito, Yasuyuki Okamura, 2007: Long-range propagation of annular beam for lidar application, Optics Communications, Vol. 279, pp. $159-167$

[9] Ziqi Peng and Tastuo Shiina, 2017: Generation control of a non-diffractive beam in random media by adjusting concentration, Optics Communications, Vol. 391, pp. 94-99 